\title{
Embarazo y riesgo posterior de enfermedades crónicas
}

Kaaja R y col. JAMA. 2005 Dec 7;294(21):2751-7.

\section{Objetivo}

Resumir la evidencia sobre la asociación entre algunas enfermedades que aparecen durante el embarazo y el riesgo posterior de presentar enfermedades.

\section{Fuente de datos}

MEDLINE: artículos en inglés de 1990 a 2005. Búsqueda manual y consulta a sitios web de sociedades científicas.

\section{Selección de estudios}

Revisión y conclusiones por consenso entre los dos autores.

\section{Resultados}

Los cambios fisiológicos adaptativos que se producen en el embarazo son:

- Aumento del $50 \%$ del volumen plasmático sin incremento eritrocitario.

- Vasodilatación por menor respuesta a la Angiotensina II y modificaciones del sistema "óxido nítrico/prostaciclinas".

- Hipercoagulabilidad por incremento de factores protrombóticos (Factor VIII, von Willebrand) y de los inhibidores de la activación del plasminógeno (PAl 1 y 2).

- Aumento de la resistencia a la insulina $(\mathrm{RI})$ al final del embarazo a causa de las hormonas placentarias.

- Alteraciones en la inmunidad celular debido al cambio en las poblaciones de linfocitos T "Helper" 1 y 2.

Sobreviene diabetes gestacional (DG) en 5\% de las embarazadas; mientras que en los diez años posteriores al embarazo, $40 \%$ de las mujeres con DG desarrollará diabetes tipo 2 (la mayoría a los cinco, alcanzándose a los diez una meseta) y $27 \%$, intolerancia a los hidratos de carbono. En todas las mujeres que presentaron DG se recomienda realizar una prueba de tolerancia oral a la glucosa entre la sexta y octava semana post parto, repitiéndola cada tres años.

La preeclampsia se asocia con elementos del síndrome metabólico (RI, hipertensión arterial, bajo colesterol HDL, hipertriglicéridemia e hiperuricemia) habiéndose documentado mayores concentraciones de adhesinas proinflamatorias y factores protrom- bóticos hasta 20 años después del parto en mujeres con historia de preeclampsia. Esta revelaría factores de riesgo preexistentes para enfermedad cardiovascular por lo que se sugiere su rastreo e intervenciones preventivas precoces.

El riesgo de trombosis venosa durante el embarazo es 7 a 10 veces mayor que fuera del mismo, pudiendo ser el tromboembolismo la primera manifestación de trombofilia hereditaria. Por ejemplo las pacientes con mutación heterocigota para el factor $V$ Leiden tienen un OR 8,3 (IC95\% 5,4-12,7) para presentar tromboembolismo y las homocigotas 34,4 (IC95\% 9,8-120) incrementándose con la edad la penetrancia de la trombofilia hereditaria, por lo que se recomienda implementar medidas de prevención.

El predominio relativo de linfocitos Th2 respecto de los Th1 es responsable de la mejoría que se observa en las enfermedades autoinmunes con predominio de estos últimos linfocitos-artritis reumatoidea, esclerosis multiple, tiroiditis- durante el embarazo y del empeoramiento de aquellas que dependen de los Th2 como el lupus eritematoso sistémico. Sin embargo la mejoría transitoria es seguida de una recaída posterior a la gestación.

El incremento en el volumen plasmático y al aumento del volumen de filtración glomerular que se observan durante el embarazo se relacionan con tres situaciones clínicas diferentes: 1) pueden enmascarar una glomerulopatía ya que la hipertensión arterial y la proteinuria características de estas enfermedades pueden ser atribuídas a preeclampsia, por lo que se recomienda el estudio de la función renal una vez finalizado el embarazo; 2) la hipervolemia y el aumento de la presión del pulso aumentan el riesgo de ruptura de aneurismas de las arterias cerebrales, renales o esplénicas; 3 ) la hipervolemia podría estar relacionada con el inicio de la miocardiopatía periparto, una miocardiopatía dilatada de etio-logía incierta que aparece al final del embarazo y se presenta con una frecuencia de $1 / 1300$ a 1/15000 embarazos.

En $1 \%$ de los embarazos aparece colestasis intrahepática, que se asocia con aumento de la frecuencia de parto pretérmino y muerte fetal en las últimas semanas de la gestación. Un $22 \%$ de las mujeres que presentan este síndrome desarrollarán litiasis biliar por lo que sería aconsejable controlarlas luego del embarazo.

\section{Comentario}

El embarazo se acompaña de cambios fisiológicos ${ }^{1}$ gracias a los cuales el organismo de la madre, además de aceptar un organismo genéticamente diferente, lo nutre hasta que alcanza la madurez necesaria para vivir separado de ella ${ }^{2}$. Estos cambios incluyen aumento de la volemia y de la resistencia a la insulina ${ }^{3}$; así como modificaciones en la reactividad vascular, inmunidad celular y mecanismos de la hemostasia.

Sin embargo, estos cambios pueden interactuar con situaciones genéticas 0 adquiridas para desencadenar problemas de salud durante la gestación como preeclampsia o diabetes gestacional o desenmascarar la propensión de presentar a lo largo de la vida enfermedades crónicas.

\section{Conclusiones del comentador}

La relación entre diabetes gestacional y diabetes no insulino dependiente está claramente demostrada, así como la asociación entre preeclampsia y diabetes. Lo mismo ocurre con la asociación entre trombofilias y fenómenos tromboembólicos, y entre colestasis intrahepática y colelitiasis. Sin embargo no están tan demostradas la asociación entre preeclampsia y síndrome metabólico, así como la de hipervolemia y cardiomiopatía periparto.

El estudio de las enfermedades clínicas durante el embarazo es un campo relativamente nuevo en la medicina ${ }^{4}$, siendo necesario profundizar los estudios en estos temas para poder producir recomendaciones basadas en la evidencia.

Raul Mejia [ Especialista en Medicina Interna. Hospital de Clínicas José de San Martín. ]

Mejía R. Embarazo y riesgo posterior de enfermedades crónicas. Evid. actual. práct. ambul. 9(3);73. May-Jun. 2006.Comentado de: Kaaja RJ, Greer IA. Manifestations of chronic disease during pregnancy. JAMA. 2005 Dec 7;294(21):2751-7. PMID 16333011.

\section{Bibliografía}

1. Haas J, Jackson R, Fuentes-Afflick E, Stewart A, Dean M, Brawarsky P, Escobar G. Changes in the Health Status of Women During and After Pregnancy. J GEN INTERN MED 2004; 20:45-51.

2. De Suite M. ed Medical disorders in obstetric practice. 3rd ed, Oxford, England: Blackwell Science 1995

3. Brody SC, Harris RP, Lohr KN. Screening for gestational diabetes: a summary of the evidence for the U.S. Preventive Services Task Force. Obstet Gynecol. 2003; 101: 380-392.

4. Wilson B, Watson MS, Prescott G, Sunderland S, Campbell D, Hannaford P, Smith W. Hypertensive diseases of pregnancy and risk of hypertension and stroke in later life: results from cohort study. BMJ 2003;326:845-51. 